\title{
GROWTH RATES FOR BLOW-UP SOLUTIONS OF NONLINEAR VOLTERRA EQUATIONS
}

\author{
By \\ CATHERINE A. ROBERTS (Northern Arizona University, Flagstaff, AZ)
}

AND

W. E. OLMSTEAD (Northwestern University, Evanston, IL)

\begin{abstract}
An investigation is made of the blow-up growth property of the solution to certain nonlinear Volterra integral equations which model explosive behavior in a diffusive medium. The basic results provide the asymptotic form of the blow-up solution for a large class of kernels as well as various nonlinearities based on examples from solid combustion and adiabatic shear band formation.
\end{abstract}

1. Introduction. In [8], we considered the class of Volterra equations

$$
u(t)=\int_{t_{0}}^{t} k(t-s) G[u(s), s] d s, \quad t \geq t_{0},
$$

where the nonlinearity has the form

$$
G[u(t), t]=r(t) g[u(t)+h(t)] .
$$

That investigation was motivated by certain models of explosive behavior in a diffusive medium such as those explored in [2], [3], [6], and [7]. Consistent with those models, we assumed that the nonlinearity had the properties

$$
g(u)>0, \quad g^{\prime}(u)>0, \quad u>0,
$$

while the kernel had the properties

$$
k(t-s) \geq 0, \quad k^{\prime}(t-s)<0, \quad t>s \geq t_{0} .
$$

The given functions $r(t)$ and $h(t)$ were required to be continuously differentiable and

$$
r(t)>0, \quad h(t)>0, \quad t \geq t_{0} .
$$

These functions can enhance the nonlinear growth in (1.2), which is in competition with the diffusive decay of the kernel (1.4). With the additional constraints that $g^{\prime \prime}(u)>0$ and that $r(t)$ and $h(t)$ be nondecreasing, the principal results of [8] provide criteria for the solution of (1.1) to experience blow-up. Those criteria provide 
that the monotonically increasing solution of (1.1) loses its continuity at some $\hat{t} \leq$ $t^{* *}<\infty$ whenever there exists a finite $t^{* *}$ such that

$$
\int_{t_{0}}^{t^{* *}} k\left(t^{* *}-s\right) r(s) d s=\int_{h\left(t_{0}\right)}^{\infty} \frac{d u}{g(u)}<\infty .
$$

The issue of the growth property of the solution $u(t)$ near blow-up was addressed in [8] for only one specific kernel.

The purpose of this investigation is to explore the blow-up growth property of the solution to (1.1) under more general circumstances. Our approach here will focus on the situation in which blow-up does indeed occur. That is, (1.1) has a unique solution and

$$
u(t) \rightarrow \infty \text { as } t \rightarrow \hat{t}<\infty .
$$

Based upon this assumption, we will develop a self-consistent asymptotic analysis of (1.1) which will determine the leading-order behavior of $u(t)$ as $t \rightarrow \hat{t}$ for a large class of kernels and various nonlinearities.

For convenience, we will assume here that the properties (1.3)-(1.5) hold; however, it will become evident that our analysis can apply under other, less restrictive, circumstances. It should also be pointed out that our analysis does not determine the explicit value of the blow-up point $\hat{t}$. Other methods, such as the bounds provided in [8], are required to locate $\hat{t}$, as well as to guarantee its existence.

In the analysis to follow, we will assume that the nonlinearity $g(u)$ has the asymptotic behavior

$$
g(u) \sim u^{m}(\log u)^{-n} \exp \left(u^{p}\right) \text { as } u \rightarrow \infty,
$$

where $m, n, p \geq 0$. The kernel is assumed to have the asymptotic behavior

$$
k(t-s) \sim k_{0}(t-s) \equiv \frac{1}{\Gamma(\mu)}(t-s)^{\mu-1}, \quad 0<\mu<1, \text { as } s \rightarrow t,
$$

where $\Gamma(\mu)$ is the Gamma function. These asymptotic properties are consistent with (1.3) and (1.4), respectively. Our results will be valid for a range of values of the parameters $m, n$, and $p$.

2. Asymptotic analysis. Under the assumption that the solution of (1.1) satisfies (1.7), we introduce the transformation

$$
\eta=(\hat{t}-t)^{-1}-\eta_{0}, \quad \eta_{0}=\left(\hat{t}-t_{0}\right)^{-1}, \quad w(\eta)=u(t) .
$$

Thus, blow-up condition (1.7) is now expressed as

$$
w(\eta) \rightarrow \infty \quad \text { as } \eta \rightarrow \infty .
$$

Under the transformation (2.1), the integral equation (1.1) now takes the form

$$
w(\eta)=\int_{0}^{\eta} k\left\{(\eta-\xi)\left[\left(\xi+\eta_{0}\right)\left(\eta+\eta_{0}\right)\right]^{-1}\right\} \Phi(\xi) d \xi, \quad \eta \geq 0,
$$

where

$$
\Phi(\eta)=\left(\eta+\eta_{0}\right)^{-2} r\left[\hat{t}-\left(\eta+\eta_{0}\right)^{-1}\right] g\left\{w(\eta)+h\left[\hat{t}-\left(\eta+\eta_{0}\right)^{-1}\right]\right\}
$$

The advantage of this formulation is that certain techniques developed in [1] for the asymptotic evaluation, as $\eta \rightarrow \infty$, of integrals like that in (2.3) can be utilized. 
Those techniques have been employed effectively on related problems in [4], [5], and [7].

Following the methods of [1], let $\xi=\eta \tau$, whereupon (2.3) becomes

$$
w(\eta)=\eta I(\eta)
$$

where

$$
I(\eta)=\int_{0}^{1} k\left\{\eta(1-\tau)\left[\left(\eta \tau+\eta_{0}\right)\left(\eta+\eta_{0}\right)\right]^{-1}\right\} \Phi(\eta \tau) d \tau .
$$

Since our interest is in an asymptotic solution of (2.5) as $\eta \rightarrow \infty$, we will utilize the asymptotic property (1.9) of the kernel to simplify $I(\eta)$. In (2.6) it is clear that if $\tau>0$, then the argument of the kernel is small as $\eta \rightarrow \infty$, so that $k$ can be replaced by $k_{0}$. Moreover, an estimate of the contribution near $\tau=0$ is found to be negligible. Thus we have the uniform approximation, as $\eta \rightarrow \infty$,

$$
\begin{aligned}
I(\eta) & \sim \int_{0}^{1} k_{0}\left\{\eta(1-\tau)\left[\left(\eta \tau+\eta_{0}\right)\left(\eta+\eta_{0}\right)\right]^{-1}\right\} \Phi(\eta \tau) d \tau \\
& =\left(\frac{\eta}{\eta+\eta_{0}}\right)^{\mu-1} \int_{0}^{\infty} K(\tau) F(\eta \tau) d \tau \\
& \sim \int_{0}^{\infty} K(\tau) F(\eta \tau) d \tau,
\end{aligned}
$$

where

$$
K(\tau)=\frac{1}{\Gamma(\mu)}(1-\tau)^{\mu-1} H(1-\tau), \quad F(\eta \tau)=\left(\eta \tau+\eta_{0}\right)^{1-\mu} \Phi(\eta \tau)
$$

As shown in [1], the integral over $(0, \infty)$ in $(2.7)$ can be converted to one along a vertical path in the complex plane by application of the Parseval formula for Mellin transforms. This yields

$$
I(\eta) \sim \frac{1}{2 \pi i} \int_{c-i \infty}^{c+i \infty} \mathrm{M}[K(\tau) ; 1-z] \mathrm{M}[F(\eta \tau) ; z] d z,
$$

where the Mellin transform operator is defined by

$$
\mathrm{M}[\nu(\tau) ; z]=\int_{0}^{\infty} \tau^{z-1} \nu(\tau) d \tau
$$

In (2.9), the vertical path of integration in the complex $z$-plane lies within the common strip of analyticity for the two Mellin transforms. Further simplication of (2.9) follows from noting that

$$
\mathrm{M}[F(\eta \tau) ; z]=\eta^{-z} \mathrm{M}[F(\tau) ; z],
$$

and

$$
\mathrm{M}[K(\tau) ; 1-z]=\frac{1}{\Gamma(\mu)} \int_{0}^{1} \tau^{z-1}(1-\tau)^{\mu-1} d \tau=\frac{\Gamma(1-z)}{\Gamma(1+\mu-z)} .
$$

This allows us to replace $(2.5)$ by the asymptotic equation

$$
w(\eta) \sim \eta \frac{1}{2 \pi i} \int_{c-i \infty}^{c+i \infty} \eta^{-z} \frac{\Gamma(1-z)}{\Gamma(1+\mu-z)} \mathbf{M}[F(\tau) ; z] d z, \quad \eta \rightarrow \infty .
$$


In order to continue with the analysis of (2.13), we must examine the properties of $\mathrm{M}[F ; z]$. These properties are determined by the asymptotic behavior of $F(\eta)$ as $\eta \rightarrow \infty$, where

$$
F(\eta)=\left(\eta+\eta_{0}\right)^{-1-\mu} r\left[\hat{t}-\left(\eta+\eta_{0}\right)^{-1}\right] g\left\{w(\eta)+h\left[\hat{t}-\left(\eta+\eta_{0}\right)^{-1}\right]\right\} .
$$

Before proceeding with the blow-up analysis, some discussion is in order about the situation in which $\hat{t}$ is not a point of blow-up. Since our analysis is independent of any explicit designation of $\hat{t}$, we must be cognizant of the possibility that $\hat{t}$ might not represent a point of blow-up. Even though this paper is concerned with the case when the solution does blow-up, our analysis thus far must also be valid for the case when there is not a blow-up at $\hat{t}$. We can easily account for the case when $\hat{t}$ is not a blow-up point by demonstrating that (2.13) is self-consistent with the asymptotic behavior that the solution approaches a finite constant value. That is, if $\hat{t}$ is not a blow-up point, then

$$
w(\eta) \sim A \text { as } \eta \rightarrow \infty .
$$

When (2.15) applies, then (2.14) yields

$$
F(\eta) \sim r(\hat{t}) g[A+h(\hat{t})] \eta^{-1-\mu} \quad \text { as } \eta \rightarrow \infty .
$$

It then follows from [1] that $\mathrm{M}[F ; z]$ has a simple pole at $z=\mu+1$. Since $\mu>0$, this pole does not provide the dominant contribution in the integral in (2.13) because $\Gamma(1-z)$ has a pole at $z=1$. Given that

$$
\Gamma(1-z) \sim-(z-1)^{-1} \text { as } z \rightarrow 1,
$$

then the leading asymptotic contribution from the integral in (2.13) comes from displacing the vertical contour path to the right of this pole. Thus, the asymptotic equation (2.13) takes the form

$$
A \sim \frac{1}{\Gamma(\mu)} \mathrm{M}[F ; 1]=\frac{1}{\Gamma(\mu)} \int_{0}^{\infty} F(\eta) d \eta .
$$

This demonstrates the self-consistent balance of constant terms when $\hat{t}$ is not a point of blow-up and hence (2.15) applies. An explicit determination of $A$ from (2.18) does not readily follow.

To return to the case in which blow-up does occur at $\hat{t}$, then (2.2) applies and (2.13) must provide a self-consistent balance for that situation. For the asymptotic behavior of the nonlinearity in (1.1) as given by (1.8), it follows that

$$
F(\eta) \sim r(\hat{t}) \eta^{-1-\mu} w^{m}(\eta)[\log w(\eta)]^{-n} \exp \left[w^{p}(\eta)\right] \quad \text { as } \eta \rightarrow \infty .
$$

We will examine various special cases of the nonlinear dependence in (2.19). The first case we consider is that for an algebraic nonlinearity,

$$
F(\eta) \sim r(\hat{t}) \eta^{-1-\mu} w^{m}(\eta), \quad m>1 \quad \text { as } \eta \rightarrow \infty .
$$

For this case, we assume

$$
w(\eta) \sim A \eta^{\prime}, \quad l>0 \quad \text { as } \eta \rightarrow \infty,
$$


where $A$ and $l$ are to be determined. It follows that

$$
F(\eta) \sim r(\hat{t}) A^{m} \eta^{-1-\mu+m l} \text { as } \eta \rightarrow \infty .
$$

With the restriction $1+\mu-m l<1$, it follows that $\mathrm{M}[F ; z]$ has a simple pole at $z=1+\mu-m l$ and

$$
\mathrm{M}[F ; z] \sim-\frac{r(\hat{t}) A^{m}}{z-(1+\mu-m l)} \text { as } z \rightarrow 1+\mu-m l<1 .
$$

Now the leading asymptotic contribution from the integral in (2.13) comes from the pole implied by (2.23). As the vertical path is displaced to the right, this pole is encountered before the pole implied by (2.17). Thus, (2.13) takes the form

$$
A \eta^{l} \sim \frac{r(\hat{t}) A^{m} \Gamma(m l-\mu)}{\Gamma(m l)} \eta^{m l-\mu} \text { as } \eta \rightarrow \infty .
$$

From (2.24) we deduce that

$$
l=\frac{\mu}{m-1}, \quad A=\left[\frac{\Gamma\left(\frac{m \mu}{m-1}\right)}{r(\hat{t}) \Gamma\left(\frac{\mu}{m-1}\right)}\right]^{\frac{1}{m-1}}, \quad m>1 .
$$

The next case we consider from (2.19) will include both an algebraic and a logarithmic nonlinearity. That is,

$$
F(\eta) \sim r(\hat{t}) \eta^{-1-\mu} w^{m}(\eta)[\log w(\eta)]^{-n}, \quad m>1, n>0 \quad \text { as } \eta \rightarrow \infty .
$$

For this case, we assume

$$
w(\eta) \sim A \eta^{l}(\log \eta)^{k}, \quad l>0 \quad \text { as } \eta \rightarrow \infty,
$$

where $A, l$, and $k$ are to be determined. It follows that

$$
\begin{aligned}
F(\eta) & \sim r(\hat{t}) A^{m} \eta^{-1-\mu+m l}(\log \eta)^{k m}[l \log \eta+k \log (\log \eta)+\log A]^{-n} \\
& \sim r(\hat{t}) A^{m} l^{-n} \eta^{-1-\mu+m l}(\log \eta)^{k m-n} \quad \text { as } \eta \rightarrow \infty .
\end{aligned}
$$

With the restriction that $1+\mu-m l<1$ and $k m-n=N=1,2, \ldots$, it follows that $\mathrm{M}[F ; z]$ has a pole of order $N+1$ at $z=1+\mu-m l$ and

$$
\mathrm{M}[F ; z] \sim \frac{(-1)^{N+1} r(\hat{t}) A^{m} l^{-n} N !}{[z-(1+\mu-m l)]^{N+1}} \quad \text { as } z \rightarrow 1+\mu-m l<1 .
$$

As in the previous case, the leading asymptotic contribution from the integral in (2.13) comes from the pole implied by (2.29). Thus, (2.13) takes the form

$$
A \eta^{l}(\log \eta)^{k} \sim \frac{r(\hat{t}) A^{m} \Gamma(m l-\mu)}{l^{n} \Gamma(m l)} \eta^{m l-\mu}(\log \eta)^{k m-n} \quad \text { as } \eta \rightarrow \infty .
$$

From (2.30), it follows that

$$
\begin{gathered}
l=\frac{\mu}{m-1}, \quad k=\frac{n}{m-1}=1,2, \ldots, \\
A=\left[\frac{\mu^{n} \Gamma\left(\frac{\mu m}{m-1}\right)}{(m-1)^{n} r(\hat{t}) \Gamma\left(\frac{\mu}{m-1}\right)}\right]^{\frac{1}{m-1}}, \quad m>1, n>0 .
\end{gathered}
$$


The final case we consider from (2.19) will include both an algebraic and an exponential nonlinearity. That is,

$$
F(\eta) \sim r(\hat{t}) \eta^{-1-\mu} w^{m}(\eta) \exp \left[w^{p}(\eta)\right], \quad m \geq 0, p>0 \quad \text { as } \eta \rightarrow \infty .
$$

For this case, we assume

$$
w(\eta) \sim\left[\log \left(A \eta^{l}\right)\right]^{1 / p} \sim[l \log \eta+\log A]^{1 / p} \quad \text { as } \eta \rightarrow \infty,
$$

where $A$ and $l$ are to be determined. It follows that

$$
\begin{aligned}
F(\eta) & \sim r(\hat{t}) A \eta^{-1-\mu+l}[l \log \eta+\log A]^{m / p} \\
& \sim r(\hat{t}) A l^{m / p} \eta^{-1-\mu+l}(\log \eta)^{m / p} \text { as } \eta \rightarrow \infty .
\end{aligned}
$$

After considering the various choices to produce an asymptotic balance in (2.13), it is found to be necessary to impose the restrictions that $\mu=l$ and $m / p=N=$ $0,1,2, \ldots$. It follows that $\mathrm{M}[F ; z]$ has a pole of order $N+1$ at $z=1+\mu-l=1$ and

$$
\mathrm{M}[F ; z] \sim \frac{(-1)^{N+1} r(\hat{t}) A l^{m / p} N !}{(z-1)^{N+1}} \text { as } z \rightarrow 1 .
$$

Now the leading asymptotic contribution from the integral in (2.13) will come from a coalescence of the poles implies by (2.17) and (2.35) at $z=1$. Thus (2.13) takes the form

$$
(l \log \eta)^{1 / p} \sim \frac{r(\hat{t}) A}{\Gamma(\mu)}(\log \eta)^{m / p+1} \quad \text { as } \eta \rightarrow \infty .
$$

From (2.36), it follows that this asymptotic match must be restricted to those cases in which $m+p=1$. Together with the restriction that $m / p=N=0,1,2, \ldots$, this requires that the nonlinearity in (2.32) must be such that

$$
m=\frac{N}{N+1}, \quad p=\frac{1}{N+1}, \quad N=0,1,2, \ldots
$$

It then follows from (3.36) that

$$
l=\mu, \quad A=\frac{\mu^{1 / p} \Gamma(\mu)}{\operatorname{pr}(\hat{t})} .
$$

Some caution must be expressed here in the utilization of the constant term associated with $A$ in expansion (2.33). In a more complete asymptotic expansion, the solution would be determined to higher orders which would introduce other constant terms in addition to that involving $\log A$ as implied by (2.33). Thus, only the logarithmic growth term in (2.34) should be retained in the leading order of the expansion.

3. Summary of results. The results of Sec. 2 can now be summarized and expressed in terms of the blow-up behavior associated with (1.1). In expressing these results, we again emphasize that it has been assumed that blow-up at some $\hat{t}$ does occur.

From (2.20), (2.21), and (2.25), it follows that if

$$
g(u) \sim u^{m}, \quad m>1 \quad \text { as } u \rightarrow \infty,
$$


then

$$
u(t) \sim\left[\frac{\Gamma\left(\frac{m \mu}{m-1}\right)}{r(\hat{t}) \Gamma\left(\frac{\mu}{m-1}\right)}\right]^{\frac{1}{m-1}}(\hat{t}-t)^{-\mu /(m-1)} \quad \text { as } t \rightarrow \hat{t} .
$$

From (2.26), (2.27), and (2.31), it follows that if

$$
g(u) \sim u^{m}(\log u)^{-n}, \quad m>1, n>0 \quad \text { as } u \rightarrow \infty,
$$

then

$$
u(t) \sim\left[\frac{\mu^{n} \Gamma\left(\frac{m \mu}{m-1}\right)}{(m-1)^{n} r(\hat{t}) \Gamma\left(\frac{\mu}{m-1}\right)}\right]^{\frac{1}{m-1}}(\hat{t}-t)^{\frac{-\mu}{m-1}}\left[\log \left(\frac{1}{\hat{t}-t}\right)\right]^{\frac{n}{m-1}} \text { as } t \rightarrow \hat{t}
$$

with the restriction that

$$
\frac{n}{m-1}=1,2, \ldots
$$

From (2.32), (2.33), and (2.36)-(2.38), it follows that if

$$
g(u) \sim u^{m} \exp \left(u^{p}\right), \quad 0 \leq m<1, p>0 \text { as } u \rightarrow \infty,
$$

then

$$
u(t) \sim\left[\mu \log \left(\frac{1}{\hat{t}-t}\right)\right]^{1 / p} \quad \text { as } t \rightarrow \hat{t}
$$

with the restriction that

$$
m=\frac{N}{N+1}, \quad p=\frac{1}{N+1}, \quad N=0,1,2, \ldots
$$

Acknowledgment. This research was supported in part under NSF grant DMS 9401016.

\section{REFERENCES}

[1] N. Bleistein and R. A. Handelsman, Asymptotic Expansion of Integrals, Holt, Rinehardt, and Winston, New York, 1975

[2] D. Glenn Lasseigne and W. E. Olmstead, Ignition of a combustible solid by convection heating, J. Appl. Math. Phys. (ZAMP) 34, 886-898 (1983)

[3] D. Glenn Lasseigne and W. E. Olmstead, Ignition or nonignition of a combustible solid with marginal heating, Quart. Appl. Math. 43, 303-312 (1981)

[4] W. E. Olmstead and R. A. Handelsman, Asymptotic solution to a class of nonlinear Volterra integral equations, SIAM J. Appl. Math. 22, 373-384 (1972)

[5] W. E. Olmstead and R. A. Handelsman, Asymptotic solution to a class of nonlinear Volterra integral equations. II, SIAM J. Appl. Math. 30, 180-189 (1976)

[6] W. E. Olmstead, S. Nemat-Nasser, and L. Ni, Shear bands as surfaces of discontinuity, J. Mech. Phys. Solids 42, 697-709 (1994)

[7] W. E. Olmstead, Ignition of a combustible half-space, SIAM J. Appl. Math. 43, 1-15 (1983)

[8] Catherine A. Roberts, D. Glenn Lasseigne, and W. E. Olmstead, Volterra equations which model explosion in a diffusive medium, J. Integral Equations Appl. 5, 531-546 (1993) 